\title{
Questes
}

Revue pluridisciplinaire d'études médiévales

\section{Le Récit de la bataille de Nicopolis (1396) dans les Chroniques de Jean Froissart : de l'échec à la gloire}

\author{
Marie-Gaëtane Martenet
}

\section{OpenEdition}

Journals

Édition électronique

URL : http://journals.openedition.org/questes/4261

DOI : 10.4000/questes.4261

ISSN : 2109-9472

Éditeur

Les Amis de Questes

Édition imprimée

Date de publication : 30 octobre 2015

Pagination : 125-139

ISSN : 2102-7188

\section{Référence électronique}

Marie-Gaëtane Martenet, « Le Récit de la bataille de Nicopolis (1396) dans les Chroniques de Jean

Froissart : de l'échec à la gloire », Questes [En ligne], 30 | 2015, mis en ligne le 09 novembre 2015, consulté le 19 avril 2019. URL : http://journals.openedition.org/questes/4261 ; DOI : 10.4000/ questes.4261 


\title{
Le Récit de la bataille de Nicopolis (1396) dans les Chroniques de Jean Froissart : de l'échec à la gloire
}

\author{
Marie-Gaëtane MARTENET \\ Université Paris-Sorbonne (Paris-IV)
}

Nous sommes en juin 1395, sous le règne de Charles VI, roi à la santé fragile, dont le conseil de régence est présidé par son oncle Philippe, duc de Bourgogne. Une ambassade du roi Sigismond de Hongrie arrive à Paris pour informer la cour des intentions belliqueuses du sultan turc Bajazet, qui projette d'anéantir la Chrétienté. Le duc de Bourgogne, qui profite de l'affaiblissement de l'État royal et cherche à s'affirmer comme le chef d'un duché indépendant et non plus comme un grand vassal du roi de France, saisit cette opportunité guerrière pour tenter d'augmenter le prestige de sa maison. Il nomme son fils aîné, Jean, (le comte de Nevers, futur «Jean Sans Peur », alors âgé de 24 ans), chef de l'expédition qui sera composée de chevaliers de plusieurs nationalités. Cette nomination est un fin calcul politique, comme le souligne Jim Magee :

Si la croisade était une réussite, Jean de Nevers serait un héros; si elle était un échec, il ne deviendrait pas impopulaire (on croyait, en effet, qu'il était plus important de prendre part au mouvement de la croisade que de gagner la victoire) ; en 1396, Jean était très jeune : on ne lui attribuerait pas la défaite ; enfin, s'il était tué, 
la maison de Bourgogne aurait un martyr, et ceci rehausserait la réputation du duché ${ }^{1}$.

Au printemps de l'année 1396, l'expédition s'organise. Philippe le Hardi orchestre une véritable démonstration visuelle de la richesse de son duché, grâce à l'armement et à l'équipement qu'il fournit. Le nombre de ces chevaliers est discuté : Jean Froissart ${ }^{2}$ en compte mille tandis qu'Aziz Suryal Atiya estime à plus de dix mille le nombre d'hommes partis en Hongrie $^{3}$. Le contingent franco-bourguignon rejoint Sigismond de Hongrie à Buda, au mois de juillet 1396. Après quelques victoires sur des avant-postes turcs, les croisés visent Nicopolis, une ville fortifiée, verrou défensif turc.

Le 10 septembre, les croisés débutent le siège, sans réaliser que Bajazet, parfaitement informé des mouvements des troupes croisées, est déjà en route pour en découdre. Il arrive le 24 septembre. La bataille se déroule le lendemain le 25 septembre 1396. Les chevaliers français, bouillonnants, chargent les premiers, laissant les Hongrois de Sigismond en retrait. À ce moment, des pieux camouflés empalent les chevaux des Français qui, démontés, dans une rage indescriptible, enfoncent à pieds toute l'infanterie turque qui se débande. Les Français, ivres de vengeance, les pourchassent sur plusieurs centaines de mètres, sans réaliser qu'ils s'isolent des autres croisés. Après avoir gravi une colline, ils découvrent le restant de l'armée de Bajazet et ses renforts serbes. Il est trop tard pour une intervention de Sigismond, qui s'enfuit. Les Français sont mis en pièces ou capturés. Certains chevaliers de haute naissance ont la vie sauve, mais beaucoup de prisonniers sont exécutés le lendemain.

\footnotetext{
${ }^{1}$ Jim Magee, «Le Temps de la croisade bourguignonne : l'expédition de Nicopolis », Nicopolis 1396-1996, dir. Jacques Paviot et Martine Chauney-Bouillot, Dijon, Société des Annales de Bourgogne, 1997, p. 55.

2 Jean Froissart, Chroniques, Paris, Librairie générale française, coll. « Le livre de poche. Lettres gothiques », 2004, p. 552.

${ }^{3}$ Aziz Suryal Atiya, The Crusade of Nicopolis [1934], Londres, Methuen \& co., 1978, p. 50.
} 
Un messager retourne en France pour annoncer la défaite du comte de Nevers et débuter les négociations au sujet de la rançon des seigneurs, emmenés en Anatolie par leurs geôliers. Après plusieurs mois de tractations, sept mille florins d'or sont versés pour la libération d'Henri de Bar, de Jacques de Bourbon et du comte de Nevers. Enguerrand de Coucy, Guy de la Trémoïlle et Philippe d'Artois sont morts durant leur captivité.

Les prisonniers arrivent à Venise en octobre 1397. Enfin, grâce à l'activité du marchand Dino Rapondi, le comte de Nevers peut regagner la France en janvier 1398. Il entre triomphalement à Dijon le 23 février, salue le roi à Paris le 10, est à Arras le 17 pour saluer sa mère, le 22 à Gand pour saluer son père ${ }^{4}$. L'entreprise de glorification de la «dernière croisade » peut commencer.

\section{L'entreprise de glorification en marche}

Grâce à l'œuvre de Jean Froissart, la défaite de Nicopolis a vite revêtu un aspect mythique, pour devenir une sorte d'élément fondateur du pouvoir et du prestige de la cour de Bourgogne. Froissart, rappelons-le, est originaire de Valenciennes, où il naît vers 1337. Ses principaux protecteurs sont Jean et Philippa de Hainaut, puis Wenceslas et Jeanne de Brabant, mais aussi Gaston Fébus, comte de Foix. Ses Chroniques, contenues en quatre livres, rappellent les différents événements historiques en France et en Europe, de 1322 à 1400.

Entrons maintenant dans l'élaboration du mythe de cette défaite. Le comte Guillaume d'Ostrevant demande à son père, le duc Aubert de Bavière, l'autorisation de partir guerroyer aux côtés des Français.

\footnotetext{
${ }^{4}$ Jacques Paviot, Les Ducs de Bourgogne, la croisade et l'Orient : fin XIV siècle$X V^{e}$ siècle, Paris, Presses de l'Université de Paris-Sorbonne, coll. «Cultures et civilisations médiévales », 2003, p. 49.
} 
Froissart se fait le témoin de la mise en garde du duc, qui refuse en répondant :

Guillaume, puis que tu as la voulenté de voiagier et d'aller en Honguerie et en Turquie et querir les armes sur gens et pays qui ne nous ont jamais rien fait, ne nul title de raison ty n'y as d'y aller, fors que pour la vainne gloire de ce monde, laisse Jehan de Bourgoingne et nos cousins de France faire leurs emprises et fay la tienne à par toy ${ }^{5}$.

Autre effet d'annonce : les chrétiens ne se comportent pas comme des chevaliers. Froissart s'appesantit sur leurs exactions à Comete, une des villes conquises avant Nicopolis: «La cité par force de siege fu prinse et destruite et y ot grant occicion d'ommes, de femmes et d'enffans et n'en avoient les chrestiens qui dedens entrerent nulle pitié ${ }^{6}$. L'échec final est dès lors compris comme une conséquence d'une mauvaise conduite générale de l'armée croisée, qui s'éloigne des préceptes chevaleresques, et s'expose donc à la vengeance de Dieu offensé.

Froissart va également insister sur le fait que les préparatifs trop fastueux de l'expédition tranchent avec l'esprit affiché de croisade spirituelle, pour la Chrétienté. La croisade sert ici les intérêts politiques du duc, ainsi que le besoin d'illustration de sa famille et de sa noblesse. Le duc se détournant alors de du vrai but de la croisade : la gloire de Dieu. Le comportement profane des chevaliers est un début d'échec. Ce que condamne Froissart, ce sont les préparatifs grandioses de la maison de Bourgogne, décrits ainsi :

Semblemment tous autres barrons, chevaliers et escuiers allans soubz et en la compaignie du dit Jehan de Bourgogne s'efforcoient moult fort de eulx agenser et mettre en point chacun en son

\footnotetext{
${ }^{5}$ Jean Froissart, Chroniques, op. cit., p. 535.

${ }^{6}$ Ibid., p. 554.
} 
endroit le plus richement et au mieulx que faire le povoient ${ }^{7}$.

Un autre élément condamnable est la trop grande vanité des princes, décrits par Froissart comme tous pressés d'«advanchier en honneur ». Ce passage des Chroniques s'avère donc, comme le souligne Marie-Thérèse de Medeiros,

un fidèle miroir de la société chevaleresque de la fin du XIV siècle. Reflet ici de la modification qui s'opère dans les mentalités, toujours marquées par les valeurs du passé, et donc toutes acquises à l'idée de croisade, mais gagnées par un esprit laïc qui fait une large place aux biens de ce monde, que cela se manifeste par la primauté de la raison d'État, par la solidarité du lignage ou par le désir de faire son chemin dans la société des hommes ${ }^{8}$.

Il s'agit en effet de montrer comment les préparatifs de cette croisade ont quelque chose de profane, de superficiel et surtout d'orgueilleux. Le chroniqueur rapproche alors le comportement des chrétiens de celui de leurs ennemis sarrasins. Froissart veut montrer que la défaite est due à la mauvaise conduite de certains chrétiens. En effet, alors que le roi de Hongrie a fait mander par un de ses maréchaux de ne pas attaquer les Turcs tout de suite, les Français agissent tout autrement, et ce par orgueil. Le roi de Hongrie les met en garde ainsi :

Vous prie et mande par moy que point que vous ne fachiés si graut oultrage que vous allés commenchier la bataille et assaillir les ennemis jusques à temps que vous aurez de par le roy autres nouvelles 9 .

\footnotetext{
${ }^{7}$ Ibid., p. 224.

${ }^{8}$ Marie-Thérèse de Medeiros, Hommes, terres et histoire des confins. Les marges méridionales et orientales de la chrétienté dans les Chroniques de Froissart, Paris, Champion, coll. «Essais sur le Moyen Âge », 2003, p. 283.

${ }^{9}$ Jean Froissart, Chroniques, op. cit., p. 611.
} 
Mais, dans le camp français a lieu une querelle personnelle entre le seigneur de Coucy et Philippe d'Artois, comte d'Eu, au sujet de la stratégie à adopter. Froissart, fervent défenseur de la mesure et de la décision réfléchie, témoigne dès lors dans son récit d'un certain mépris pour le sort des Français, qui, selon lui, méritent ce qui leur arrive :

Là estoient ces seigneurs de France si richement en leurs armures et en si bel arroy que chascun sambloit ung roy [...]. Or, regardez la grant folie et le grant oultrage, car se ilz euissent attendu le roy de Honguerie et les Hongres, ou bien avoit $. l x .{ }^{\mathrm{m}}$ hommes, ilz euissent fait ung grand fait. Et par eulx et par leur orgueil fut toute la perte et le dommaige que ilz rechuprent si grant, que depuis la bataille de Rainchevaulx, où les .xii. peers de France furent mors et desconfis, ne rechuprent si grant dommaige ${ }^{10}$.

Froissart rapporte que les Hongrois demandent à leur roi la permission de quitter le champ de bataille :

Nous perderons hui la journée par l'orgueil et le beubant de ces Franchois.

Sire, sauvez vous ! car se vous estes ne mors ne pris, toute Honguerie est perdue. Il nous faut huy perdre la journée par l'orgueil des Franchois ${ }^{11}$.

Froissart achève enfin son récit par les conséquences désastreuses de cet orgueil, à savoir la défaite, la mort de très nombreux combattants, les pertes matérielles importantes, la prise de nombreux prisonniers et l'exécution de ces derniers :

Ce grant meschief et dommaige reçurent devant Nicolpoly en Turquie les Franchois et furent tous mort et tous prins, [...] Messire Jehan de Bourgoingne fu pris et aussi furent le conte d'Eu et le conte de la Marce, le sire de Coucy, messire Henry de Bar fu mort sus la place,

\footnotetext{
${ }^{10}$ Ibid., p. 613.

${ }^{11}$ Ibid., p. 614.
} 
messire Jehan de Vienne, messire Guy de la Trimouille, messire Bouchicault et messire Jacques de Helly. Et messire Philippe de Bar fu mort sus la place, messire Jehan de Vienne, messire Guillame de la Trimouille et son filz. [...] Sus l'espace de trois heures ceste grosse bataille fu faicte et perdy le roy de Honguerie tout son arroy entierement et toute sa vaisselle d'or et d'argent $[\ldots]^{12}$.

Froissart n'est donc pas un simple rapporteur des faits passés, il trouve une explication subtile à l'issue de cette bataille. Par sa narration et son jugement, il dépasse son propos et amène son lecteur à tirer des leçons du passé. On pourrait presque penser que le chroniqueur narre la bataille uniquement pour illustrer le danger de l'orgueil ${ }^{13}$. Le combat achevé, une foule de combattants est contrainte de gagner la rive gauche du Danube, au risque de se noyer, ou de se rendre, devenant ainsi les captifs de Bajazet.

Froissart insiste sur la tenue blanche des prisonniers :

Dont furent amenez, ainsi que tous nuds en leurs linges draps, l'un après l'autre, pluiseurs bons chevalliers et escuiers du royalme de France et d'autres nactions $[\ldots]$ il faisoit ung signe qu'ilz fuissent mors et detrenchies ${ }^{14}$.

Or, ce vêtement blanc a bien sûr une charge symbolique importante puisqu'il s'agit du vêtement caractéristique du martyr, déjà recouvert de la pureté de la sainteté qui l'attend au ciel $^{15}$. Ce choix est à relier aux propos du religieux de Saint-Denis au sujet du massacre des chrétiens de

${ }^{12}$ Ibid., p. 621.

${ }^{13}$ Philippe de Mézières donna la même interprétation à la défaite dans son Epistre lamentable et consolatoire, écrite dès 1397. Selon lui, les quatre vertus morales : règle, discipline de chevalerie, obéissance et justice, ont été remplacées par l'orgueil, la convoitise et la luxure. Voir Bertrand Schnerb, «1396, bataille de Nicopolis, un échec militaire majeur de la chrétienté latine », dans L'Histoire $d u$ monde au XV $V^{e}$ siècle, dir. Patrick Boucheron, Paris, Fayard, 2009, p. 297.

${ }^{14}$ Jean Froissart, Chroniques, op. cit., p. 622.

${ }^{15}$ Cette image de la captivité est également présente au fol. $75 \mathrm{v}$ de la Chronique d'Alexandre du manuscrit Dutuit 456 du Petit-Palais, Paris. 
Nicopolis. Celui-ci mentionne, comme le faisait Froissart, les victimes qui furent : «martirisés par l'amour Nostre Saulveur Jhesu Crist ${ }^{16}$, dont les corps n'ont pas subi de décomposition après la mort contrairement à ceux des Turcs. En effet, longtemps après le combat, les bêtes semblaient laisser intacts les corps des chrétiens tandis qu'elles s'attaquaient aux cadavres turcs. Ce fait fut considéré comme un miraculum: on affirma par là que les chrétiens morts à Nicopolis étaient en état de sainteté.

Je ne crois pas devoir passer sous silence un fait assez étonnant, qui fut regardé comme un miracle par quelques personnes, et que leur fit dire que Dieu, pour l'exaltation de la vraie foi, avait sanctionné le martyre des chrétiens et accordé à leurs âmes le salut éternel. Leurs corps conservèrent pendant treize mois toute leur fraîcheur, sans se corrompre ni s'altérer, et sans que les bêtes féroces ni les chiens osent y toucher ${ }^{17}$.

Selon Jacques Paviot, à la suite du désastre de Nicopolis, le duc de Bourgogne fit tout pour occulter l'aspect négatif de l'expédition : c'est pourquoi il organisa des entrées triomphales pour fêter le retour de son fils dans différentes villes du duché ${ }^{18}$. Par la suite, il n'y eut pas de nouvelles occasions d'expéditions durant le règne de Jean sans Peur (1404-1419); cependant cet événement resta une source de grand prestige pour ce jeune prince, qui montrera un intérêt littéraire pour l'Orient, perpétué par son fils Philippe le Bon (1419-1467), né en 1396, l'année du désastre ${ }^{19}$.

\footnotetext{
${ }^{16}$ Religieux de Saint-Denys, Chronique du religieux de Saint-Denys contenant le règne de Charles VI, 1380-1422, Paris, Paléo, 2007, p. 153.

${ }^{17}$ Ibid., p. 154.

${ }^{18}$ Jacques Paviot, Les Ducs de Bourgogne, la croisade et l'Orient, op. cit. p.51.

${ }^{19}$ Bertrand Schnerb, Jean sans Peur, le prince meurtrier, Paris, Payot \& Rivages, coll. «Biographies Payot », 2005, p. 295.
} 


\section{La réception de Froissart en Bourgogne}

Pour saisir l'importance accordée à l'expédition de Hongrie par les générations suivantes, il convient de recenser le nombre important de manuscrits des Chroniques de Froissart qui relatent cet événement. Nous remarquerons que la réception de l'œuvre de Jean Froissart en Bourgogne participe de la résurgence du souvenir chevaleresque et de la volonté de se tourner vers l'Orient. En effet, en ce sens, attachons-nous à rappeler deux événements fortement symboliques du règne de Philippe le Bon, à savoir la création de l'ordre de la Toison d'Or, en Janvier 1430, et la proclamation du Vœu du Faisan, en février $1454^{20}$.

L'étude des inventaires des bibliothèques des $\mathrm{XIV}^{\mathrm{e}}-\mathrm{XV}^{\mathrm{e}}$ siècles est capitale pour savoir grâce à qui et où est né l'engouement pour Froissart. Laëtitia le Guay, en 1998, avait déjà tiré des conclusions décisives ${ }^{21}$ : l'ouvrage de Froissart se trouve dans les bibliothèques aristocratiques de Bourgogne, d'Angleterre et de France. Les propriétaires des Chroniques de Froissart sont, entre autres, Philippe de Commynes, Jacques d'Armagnac, le roi d'Angleterre Edouard IV, Louis de Bruges, maréchal de Bourgogne, et Antoine, grand bâtard de Bourgogne. Le chroniqueur a bel et bien connu un grand succès dans les cours européennes.

Cependant, Froissart a été intégré à la culture historique française tardivement, sous le règne de Louis XI, de 1461 à 1483, grâce à la commande de Philippe de Commynes. Cette intégration tardive peut s'expliquer par la présentation trop favorable qu'il fait $\mathrm{du}$ roi d'Angleterre. Le succès français de Froissart est donc sans commune

\footnotetext{
${ }^{20} \mathrm{Au}$ cours d'un banquet qui se tint à Lille, Philippe le Bon jura devant sa cour de prendre le commandement d'une croisade pour délivrer Constantinople, conquise par les Turcs l'année précédente. Le projet ne vit jamais le jour.

${ }^{21}$ Laetitia Le Guay, Les Princes de Bourgogne lecteurs de Froissart. Les rapports entre le texte et l'image dans les manuscrits enluminés du livre IV des Chroniques, Paris/Turnhout, CNRS/Brépols, coll. « Documents, études et répertoires - Institut de recherche et d'histoire des textes », 1998, p. 97.
} 
mesure avec le succès qu'il connaît au même moment en Flandre bourguignonne. Par exemple, tous les manuscrits enluminés proposant les quatre livres des Chroniques ont été exécutés en Flandres, et tous les manuscrits du livre IV (intégrés à un ensemble ou non) sont issus des ateliers flamands (excepté le ms. Paris, BnF, fr. 2661 en grisailles et exécuté en France).

L'importance accordée à Jean Froissart est particulièrement visible à la cour de Bourgogne, que ce soit dans les bibliothèques des commanditaires prestigieux ou dans celle des ducs eux-mêmes. La librairie des ducs, où étaient conservés de véritables manuscrits d'art, était utilisée, comme le dit Thierry Delcourt, à des fins idéologiques et politiques, servant d'outil de propagande à la gloire de la dynastie ducale $^{22}$.

Afin d'achever ce tour d'horizon au sujet de l'engouement pour Froissart et pour l'Orient dans les bibliothèques nobiliaires de Bourgogne, il est important de constater, parallèlement à la commande documentée du seul livre IV des Chroniques par Philippe le Bon, qu'il existe un certain nombre de commandes partielles ${ }^{23}$. Au cours de ces dernières années ont été découverts quatre manuscrits relatant uniquement l'expédition de Hongrie. Ces quatre manuscrits sont d'une importance capitale pour comprendre la réception et l'entretien du souvenir de cette expédition de Hongrie au XV $\mathrm{XV}^{\mathrm{e}}$ siècle.

Ces manuscrits, après étude de la graphie et des initiales peintes ${ }^{24}$, sont datés des années 1460-80 et sont composés des différents chapitres

${ }^{22}$ Miniatures flamandes, 1404-1482, dir. Bernard Bousmanne et Thierry Delcourt, Paris/Bruxelles, Bibliothèque nationale de France/Bibliothèque royale de Belgique, 2011, p. 110.

${ }^{23}$ Georges Doutrepont, La Littérature française à la cour de Bourgogne [1909], Genève, Slatkine reprints, 1970, p. 426.

${ }^{24}$ La première partie des travaux a été effectuée par Laetitia Le Guay, Les Princes de Bourgogne lecteurs de Froissart, op. cit. 
concernant l'expédition de Hongrie contenus dans les Chroniques de Froissart, depuis l'arrivée de l'ambassade hongroise en France jusqu'au retour de captivité de Jean sans Peur. Sous le titre «Relation du voyage de Jean de Bourgogne en Turquie », on trouve donc les manuscrits :

- Paris, BnF, Naf. 7508 ;

- le manuscrit dit «Vuilleret » (en mains privées) ${ }^{25}$;

- le manuscrit dit «Arenberg» de la bibliothèque Newberry de Chicago (ms. Ry, 63-1625), jadis transcrit par Kervyn de Lettenhove à la fin du XIX ${ }^{\mathrm{e}}$ siècle $^{26}$;

- le manuscrit dit «Ashburnham» (localisation actuelle inconnue), jadis transcrit par Kervyn de Lettenhove à la fin du XIX ${ }^{\mathrm{e}}$ siècle $^{27}$;

- enfin, mentionnons pour information une copie plus tardive du XVII ${ }^{\text {e }}$ iècle, le ms. 3098 de la Bibliothèque municipale de Dijon, acquis en 1985.

Il est intéressant de voir que les manuscrits Naf. 7508 et Vuilleret, datés des années 1460-80, portent l'ex-libris des princes de Steenhuyse : Louis de Gruuthuse (1422-1492) et son fils Jean (c. 1445-1512). Il s'agit d'une lettre $\mathrm{W}$ à l'encre noire, le W symbolisant les deux U de Gruuthuse $^{28}$. Une troisième version du texte, le manuscrit «Arenberg» (ms. Ry 239 ; 63-1625, ff. 52-120v.) est quant à elle insérée dans une compilation de textes importants concernant uniquement la Bourgogne ducale.

${ }^{25}$ Vendu aux enchères le 22 décembre 2009, par M. Roch de Coligny, Paris, DrouotMontaigne.

${ }^{26}$ Jean Froissart, Euvres, publiées par Kervyn de Lettenhove, Bruxelles, 1867-1877, (réimp. Osnabrück, Biblio, 1967), fin du tome XV, p. 439.

${ }^{27}$ Ibid. p. 439.

${ }^{28}$ Il s'agit du manuscrit Paris, BnF, Naf. 7508 et du manuscrit dit Vuilleret, passé en vente en enchères à l'hôtel Drout en décembre 2009. 
Ces commandes partielles témoignent donc d'une volonté de garder intact le souvenir de Nicopolis notamment en séparant son récit du reste des Chroniques.

Illustrée par près d'une vingtaine d'images réparties dans sept manuscrits ${ }^{29}$, l'expédition malheureuse de Hongrie est présente de manière systématique dans les programmes iconographiques. Il devient incontestable que dans les anciens Pays-Bas méridionaux, durant la seconde moitié $\mathrm{du} \mathrm{Xv}^{\mathrm{e}}$ siècle, un lien unit le souvenir de Nicopolis, l'esprit de croisade, les princes bourguignons et leur bibliophilie. La mise en regard d'un récit et de son illustration, le choix et le traitement par l'image de passages écrits, révèlent une véritable lecture de l'œuvre exercée par les enlumineurs et par les commanditaires. Prenons l'exemple du travail de Philippe de Mazerolles dont témoignent les manuscrits de la British Library ${ }^{30}$ : il passe sous silence la bataille, donc la défaite. En effet, le conflit est sous-entendu mais jamais représenté. On observe le départ du contingent de Jean de Nevers, avec un certain nombre d'hommes en armes, ou encore le conseil des chefs d'armes européens en Hongrie, mais pas une seule enluminure ne met en scène le combat. Différentes explications sont envisageables. D'une part, on sait que cet enlumineur a un goût pour la peinture de la vie de cour et du faste dynastique: il lui semblerait donc plus adapté de représenter les événements qui entourent la bataille plutôt que le conflit en lui-même. De ce point de vue, Philippe de Mazerolles est très proche de la sensibilité du chroniqueur qui, lui aussi, préfère dépeindre les rouages du pouvoir plutôt que la bataille (le chapitre proprement dit où est évoqué le combat est maigre). À trois quarts de siècle de distance, on ressent une même

\footnotetext{
${ }^{29}$ Paris, BnF, fr. 2 646, 2 648, 2 661. Paris, Arsenal, ms. 5 190. London, British Library, Harley, 4379-80; Royal 18, II, 2. Berlin, Staatsbibliothek und Preussischer Kulturbesitz, Dépôt Breslau I, ms. Rehdiger 4.

${ }^{30}$ London, British Library, Harley, 4 379-80.
} 
adhésion de l'enlumineur et du chroniqueur aux représentations que le pouvoir donne de lui-même. On parle d'ailleurs «d'État-spectacle » ${ }^{31}$ pour caractériser la politique bourguignonne à la fin du Moyen Âge.

Etant donné l'issue de cette expédition, les commanditaires ont pris soin d'en montrer une vue d'ensemble positive. Rétrospectivement, le lecteur-spectateur doit voir une noblesse en armes, se préparant au conflit, et non des scènes rappelant l'issue néfaste de la bataille. Le lecteur n'a donc pour ainsi dire pas conscience que l'épisode relaté est celui de la débâcle de Nicopolis, car il a devant les yeux le quotidien de la noblesse, partant et rentrant de croisade sans encombre.

Philippe de Mazerolles nous offre huit enluminures de l'expédition de Hongrie $^{32}$. Ces huit enluminures peuvent être séparées en deux groupes: le premier correspondant à l'aide demandée par le roi de Hongrie, incluant l'arrivée des lettres, le départ et le conseil (ff. 54 à 84), le second correspondant au sort des captifs après la défaite (ff. 98 à $\left.131 v^{0}\right)$. On observe ainsi qu'une ellipse temporelle passe sous silence les événements ayant lieu entre le conseil tenu par Sigismond au mois de Juillet 1396 et l'annonce de la défaite au roi de France à l'automne 1396, voire en décembre. Plusieurs mois et un certain nombre d'épisodes relatés par Jean Froissart sont écartés du programme iconographique.

Cette mise sous silence présente deux aspects importants. D'une part, elle laisse en effet place à la représentation de motifs moins récurrents dans la tradition iconographique de cet épisode. Philippe de Mazerolles, toujours attiré par la peinture de l'aristocratie, préfère ainsi mettre en scène les ambassades, les conseils, les salutations ou le

${ }^{31}$ Evelyne van den Neste, Tournois, joutes et pas d'armes dans les villes de Flandres à la fin du Moyen Âge (1300-1386), Paris, Ecole Nationale des Chartes, 1996, p.202.

${ }^{32}$ London, British Library, Harley, 4 379-80 : ff. 54; 58v; 84; 98; 106; 118; 124v; $131 \mathrm{v}$. 
paiement des rançons. Sur les huit enluminures, on trouve deux ambassades, deux conseils, un paiement de rançon, deux départs du contingent européen et une arrivée à Paris. Ces enluminures ont donc une vocation politique.

D'autre part, on observe une mise à l'honneur de la Bourgogne et de la puissance financière de Philippe le Hardi. Comme on l'a vu plus haut, les origines de la croisade se situent davantage à la cour de Bourgogne qu'à la cour de France. En effet, Philippe le Hardi, à partir de 1390, se comporte en roi dans ses terres, plutôt qu'en vassal de la couronne. Il décide ainsi d'affermir son autorité en organisant des projets pour son duché. Tout cela est clairement visible dans les enluminures de Philippe de Mazerolles. Ainsi dès que le roi de France est représenté, Philippe le Hardi l'est également, affirmant son pouvoir au sein du royaume (ff. 98 et 106). Quant à Jean de Nevers, érigé en héros de l'expédition, il apparaît sur quatre enluminures (ff. $58 \mathrm{v}^{\circ}, 84,124 \mathrm{v}^{\circ}$, $\left.131 v^{0}\right)$. Une dernière enluminure peut être reliée à cette peinture du pouvoir ducal : celle du paiement de la rançon à Bajazet (fol. 118). En effet, le duc de Bourgogne est le personnage qui a fourni le plus d'argent pour la rançon. Il semblait donc important à l'enlumineur et au commanditaire de témoigner de la puissance financière du duc.

Ainsi sur les huit enluminures, sept font figurer le duc, son fils et la richesse du duché. La mise en valeur de la Maison de Bourgogne sur les images de l'expédition de Hongrie est donc évidente. On peut l'interpréter, d'une part, comme la volonté de montrer la réalité de l'expédition, à savoir qu'elle était avant tout bourguignonne et qu'elle fut source de prestige pour la nouvelle dynastie, et, d'autre part, comme la manifestation de l'orientation politique et idéologique voulue par le commanditaire, ici, Philippe de Commynes, toujours au service de Charles le Téméraire et soucieux de magnifier le pouvoir ducal. 
Ces illustrations ou ces commandes partielles témoignent du fait que Jean Froissart était fort apprécié dans le milieu anglo-bourguignon. D'autre part, elles révèlent quel regard les hommes du $X^{\mathrm{e}}$ siècle portaient sur l'œuvre du chroniqueur. La vision que ces hommes avaient de la bataille de Nicopolis mêlait le rêve au souvenir, effaçant la défaite au profit des hauts faits et présentant l'erreur humaine et l'échec comme autant de leçons à méditer. 